\title{
V479 And: CV, LMXB, or Symbiotic?
}

\section{Diego González Buitrago, Gagik Tovmassian, Juan Echevarría, Sergey Zharikov, Takamitsu Miyaji, Andres Avilés, and Gennady Valyavin}

\author{
Instituto de Astronomía, UNAM, Apdo. Postal 877, Ensenada, BC, 22800 Mexico
}

\begin{abstract}
V479 And is a 14.26 hour, close binary system, comprised of a G8-K0 star departing from the main sequence and a compact primary star accreting matter from the donor. The object is an X-ray source, modulated with the orbital period. This, and the presence of an intense He II line, leads us to speculate that the compact object is a magnetic white dwarf. However, we do not find strong constraints on the upper mass limit of the compact object, and we may have a neutron star in a low mass X-ray binary instead of a cataclysmic variable. The orbital period is certainly too short for the donor star to be an evolved giant star, so classifying this object as a symbiotic binary may be a big stretch; however there is an evidence that the mass transfer occurs via stellar winds, rather than through the $\mathrm{L}_{1}$ point of Roche filling secondary, a phenomenon more common for symbiotic stars.
\end{abstract}

Keywords. binaries: close, spectroscopic, symbiotic — stars: novae, cataclysmic variables, magnetic fields, individual (V479 And, SDSS J001856.93+345444.3) — X-rays: binaries

\section{Introduction}

V479 And, classified initially as SDSS J001856.93+345444.3, was identified as a cataclysmic variable (CV) by Szkody et al. (2005). Neither they, nor Dillon et al. (2008), could find any orbital-period-related variability. González et al. (2010) conducted an extensive spectroscopic campaign in 2008-09. A $14.52 \pm 0.53$ hour period, $P_{\text {orb }}$, was deduced by cross-correlating the absorption features in the spectra of the object with a $\mathrm{K} 2-\mathrm{K} 4 \mathrm{~V}$ standard star template. Radial velocity (RV) measurements of emission lines obtained by fitting a Gaussian did not fold with the period determined from the absorption lines. It was suggested that the object might be an asynchronous polar.

\section{Observations}

We collected additional observations of V479 And, beyond what we reported in our previous work (González et al. 2010). We obtained higher resolution spectroscopy and linear photo-polarimetry in $\mathrm{V}$ band. We also collected I-band light curves and some Jband near-IR photometry, but with a very low signal-to-noise ratio. Finally, we obtained UV and X-ray observations with the UVOT and XRT instruments on board the Swift observatory.

\section{The Components of the Binary System}

The optical spectrum of V479 And shows absorption features from the donor star, most prominently in the $\lambda 5000-6000 \AA$ range. A careful analysis of the absorption features, particularly in the $\lambda 4200-4300 \AA$ region, shows that the donor star is hotter than we previously thought and is similar to our observed standard stars of spectral class G8-K0. Unfortunately, the temperature sensitive lines of Ca I $4226 \AA$, Cr I 4254, $4290 \AA$, and Fe I $4271 \AA$ lie in a contaminated region, precluding a better identification of the spectral type. We repeated the procedure of measuring the radial velocities via cross-correlation with a 
K0 template on the extended spectral data, and obtained the following new ephemeris: $\mathrm{HJD}=2454776.34796(970)+0.5940934(131) \times \mathrm{E}$.

Although we are unable to determine the luminosity class of the donor star from the spectra, a large $P_{\text {orb }}$ implies that some evolution should exist in order to fill its corresponding Roche lobe, regardless the mass of the compact accretor. Figure 1 shows close binary systems for donor stars with known spectral types. The red line indicates the Roche lobe size of corresponding main sequence donor stars and $P_{\text {orb }}$ for a fixed compact primary mass of $0.7 \mathrm{M}_{\odot}$.

The black lines are evolutionary tracks of donors with masses $\geqslant 0.9 \mathrm{M}_{\odot}$ from the ZAMS to the subgiant branch. While it is known that secondaries in semi-detached short-period CVs deviate in size from their single counterparts by as much as $8 \%$ (Echevarria 1983, Knigge et al. 2011), the departure of secondary stars in systems with periods $>10$ hours is much more significant. The secondaries in these long period systems could have been massive enough to have started evolution before entering into contact and becoming interacting binaries. Their progenitors might have been mid-G type ZAMS stars which evolved into larger late-G/early-K subgiants with lower $T_{\text {eff }}$.

On top of the spectra, which have flat continua and numerous absorptions lines, there are relatively narrow $\left(\approx 600 \mathrm{~km} \mathrm{~s}^{-1} \mathrm{FWHM}\right)$ and intense emission lines of $\mathrm{H}$ and He. Measuring them by fitting a single Gaussian does not provide a good result, and thus González et al. (2010) wrongly interpreted these results as a deviant period. On our higher resolution spectra, it is evident that there is a broader base component to the emission lines. We used the double-Gaussian method to measure the RV of the emission lines and found no discrepancy between the period deduced from the absorption and the emission lines. The measured RVs result in a semi-amplitude of the donor of $\mathrm{K}_{\mathrm{d}}=59 \pm$ $4 \mathrm{~km} \mathrm{~s}^{-1}$ and a semi-amplitude of the accretor of either $\mathrm{K}_{\mathrm{acc}}=32 \pm 5 \mathrm{~km} \mathrm{~s}^{-1}$ (based on $\mathrm{H}_{\beta}$ ) or $42 \pm 9 \mathrm{~km} \mathrm{~s}^{-1}$ (based on He II). The $\mathrm{H}_{\beta}$ emission might be seriously affected by the absorption from the donor star, which contributes $50 \%$ of light; therefore, the higher RV measured from the He II line is a better indicator of the velocity of the accretor. It is not clear where the wings of the emission lines come from. In a classic CV, the broad emission lines are emitted by the accretion disk. Since the disk rotates with Keplerian velocities, the low velocity regions of the disk contribute to the center of the line, while the inner parts close to the white dwarf (WD) rotate faster and form the wings of the lines. However, in V479 And the central and intense part of the emission lines do not show orbital motion and has rather the observational characteristics of a polar object in which the magnetosphere of a highly magnetic WD prevents the formation of the accretion disk. The emission lines in polars are usually multicomponent: narrow emission lines (NELs) originating from the irradiated secondary star, high velocity components (HVCs) produced by the ballistic part of the mass transfer stream, and broad base components (BBCs) thought to be magnetically channeled matter (Schwope et al. 1997).

We do not detect NEL or HVC components, but rather a BBC component, consistent with a polar-like object. If such, the wings of the emission lines should be closely associated with the dwarf orbital motion, and we can safely use $K_{d}$ and $K_{\text {acc }}$ to construct an $M_{\mathrm{d}}-M_{\mathrm{wd}}$ diagram. This is shown in Figure 2. The straight diagonal lines are mass ratio $\mathrm{M}_{\mathrm{d}} / \mathrm{M}_{\mathrm{acc}}=\mathrm{K}_{\mathrm{acc}} / \mathrm{K}_{\mathrm{d}}$ solutions. The lines stemming from the He II and $\mathrm{H}_{\beta}$ measurements are marked by thicker lines $(\mathrm{q}=0.71$ and 0.54 respectively). The shaded area marks the region for the mass of a main-sequence K0 star up to a subgiant star. The accretor, within the limits of this figure, is probably a massive WD, but a neutron star is not excluded.

The photometry of the objects shows variability on some nights, reaching 0.3 mag, but the pattern of variability is not systematic. It does not repeat with $P_{\text {orb }}$, and the variability changes from epoch to epoch. As such, its behavior is reminiscent of another 
extremely long period CV: EY Cyg (Echevarría et al. 2007), and we are inclined to think that such irregular variability is connected to a spotted surface of the donor star. As for polarimetry, the object does not show any measurable linear polarization. The J-band measurements had errors too large to allow temporal analysis, but apparently there is variability in the near-IR too. A donor star with a spotted surface is an indicator of magnetic activity. It should be noted here that a strong magnetic field in a WD would not be enough to synchronize its rotation with the $P_{\text {orb }}$ in such a widely separated system. Frank et al. (1995) suggested a scenario for long period polars according to which the secondary star is also strongly magnetic, leading to a strong wind which is captured and accreted by the magnetic field of the WD. The synchronization happens due to dipole locking.

The X-ray data is too noisy to fit the spectrum unambiguously, but the X-ray light curve definitely shows one sharp peak per orbit with count rate changing on average from $0.01 \mathrm{cnts} \mathrm{s}^{-1}$ at the bottom to $0.06 \mathrm{cnts} \mathrm{s}^{-1}$ at the peak. Moreover, the UV data, taken simultaneously with the X-rays by the $S$ wift instruments, shows a similar modulation with $P_{\text {orb }}$ of the light curve. The peak in the UV lags $\approx 0.2 \mathrm{P}_{\text {orb }}$ relative to the $\mathrm{X}$-rays. Such behavior is common for polars, where accretion occurs along the field lines of a highly magnetic WD.

With the data collected from IR to X-ray, we can also consider the spectral energy distribution (SED) of the object. In the optical part of the SED, the donor star contributes at least $50 \%$ of the light as determined from the spectra. Regardless if it is a G8 or K0 or even K1, the donor star can not account for the IR excess. The remaining UV part of the SED can be perfectly fit by a $19 \mathrm{kK}$ blackbody, except for the SDSS u'-band data. But a WD of such temperature should have a size of one order larger than an ordinary WD to be consistent. Similarly, a UV and IR variable contribution in the SED of EF Eri (Szkody et al. 2006) was identified by Campbell et al. (2008) to be due to cyclotron radiation. The cyclotron emission was also a key to explain the IR excess and the variability in EF Eri.

\section{Conclusions}

We conducted a multi-wavelength study of V479 And from IR to X-rays. We confirm it is a 0.5941 -day close binary, probably consisting of a strongly magnetic and massive WD, which accretes matter captured from the stellar wind of an evolved K0 subgiant. The WD appears to be locked in synchronized rotation with the orbit, according to concurring period determinations from absorption and emission lines as well as UV/X-ray data. In order to achieve that in a 14 hour system, the donor star must also be magnetically active to provide dipole/dipole locking. In such a scenario, the UV and IR excess and variability can be explained in the terms of cyclotron emission.

The observational data do not provide strong constraints on the mass of the accretor, and in an alternative scenario it may be a neutron star. This would mean that $P_{\text {orb }}$ is not unusually long, and places it in the middle of the $P_{\text {orb }}$ distribution of low mass X-ray binaries. But it would create a big difficulty in explaining the X-ray and UV modulation with $P_{\text {orb }}$, the nature of UV emission, and IR excess. There is weak similarity of the SED of V479 And with those of microquasars in quiescence, particularly XTE J1118+480 (McClintock et al. 2003). In such a case, the UV and X-ray radiation should be probably fit by ADAF models (Narayan et al. 1996), but the amount and quality of our data is not sufficient to explore such possibilities.

We find the magnetic WD scenario with wind accretion to be a more viable solution. Of course, with a donor star barely evolving towards a subgiant, it is senseless to speak of the object as being a symbiotic binary. However, the phenomenon of a WD accreting from 


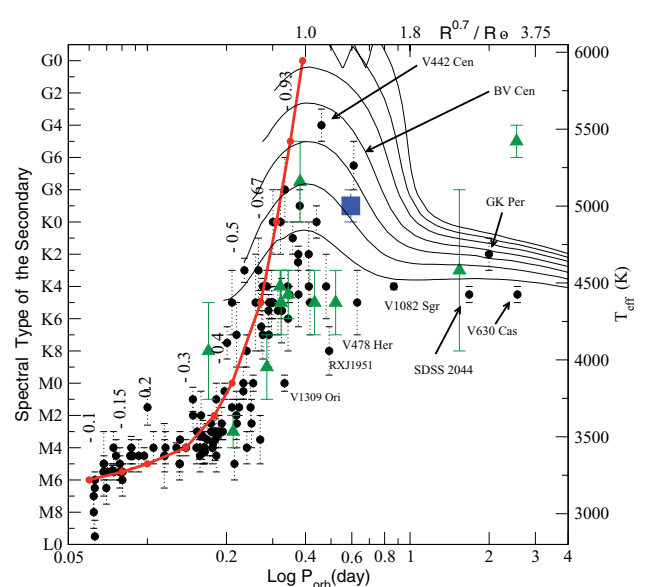

Figure 1. Orbital period vs. spectral type for CVs (circles) and LMXBs (triangles). V479 And is marked by a square. The upper $\mathrm{x}$-axis marks the Roche lobe size of the donor star (in $\mathrm{R}_{\odot}$ ) for a typical mass of the primary star of $\mathrm{M}_{1}=0.7 M_{\odot}^{\dagger}$. The thick red line corresponds to main sequence stars filling their Roche lobes. The black curves are evolutionary tracks from the ZAMS to subgiant branch stars taken from Bertelli et al. (2008).

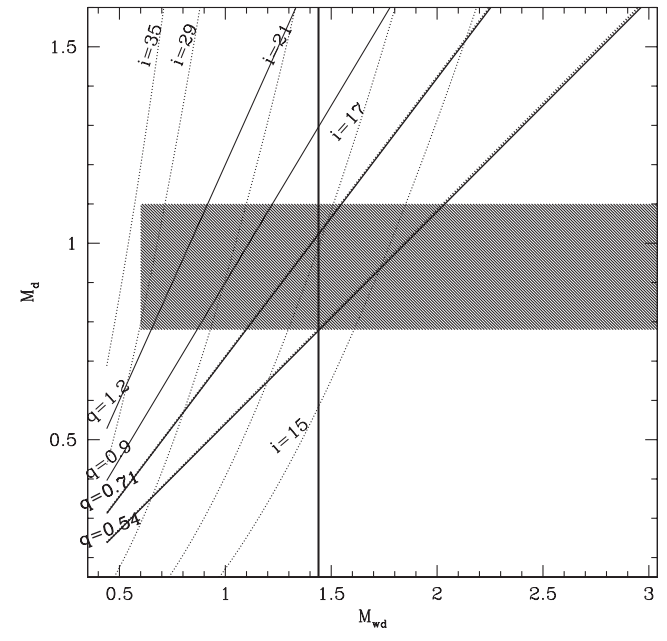

Figure 2. The $M_{\mathrm{d}}-M_{\mathrm{wd}}$ diagram for V479 And. The diagonal lines represent different mass ratios. Our best solution, considering that the measurements of the He II emission line are the best tracers of the compact star's orbital motion, is $q=0.7$. Measurements of the $\mathrm{H}_{\beta}$ line correspond to $q=0.54$. Dashed curves denote the orbital inclination of the system. The shaded area corresponds to mass range of a $\mathrm{K} 0$ star from ZAMS to luminosity class IV.

an evolved donor star via a stellar wind is more similar to symbiotics than cataclysmic variables, which traditionally are considered to be composed of a semi-detached binary where matter is transferred through the Lagrangian $\mathrm{L}_{1}$ point via a ballistic stream from a Roche lobe filling main sequence secondary. Taking into account that a WD in this system must be massive and that magnetic accretion from a wind must be more efficient than in usual symbiotics, this object is a good candidate for a Type Ia supernova.

\section{References}

Bertelli, G., Girardi, L., Marigo, P., \& Nasi, E. 2008, A\&A, 484, 815

Campbell, R. K., Harrison, T. E., Schwope, A. D., \& Howell, S. B. 2008, ApJ, 672, 531

Dillon, M., et al. 2008, MNRAS, 386, 1568

Echevarria, J. 1983, RevMexAA, 8, 109

Echevarría, J., Michel, R., Costero, R., \& Zharikov, S. 2007, A\&Ap, 462, 1069

Frank, J., Lasota, J.-P., \& Chanmugam, G. 1995, ApJ, 453, 446

González, D., Tovmassian, G., Zharikov, S., Avilés, A., \& Echevarria, J. 2010, arXiv:1009.5803

Knigge, C., Baraffe, I., \& Patterson, J. 2011, ApJ (Supp.), 194, 28

McClintock, J. E., et al. 2003, ApJ, 593, 435

Narayan, R., McClintock, J. E., \& Yi, I. 1996, ApJ, 457, 821

Schwope, A. D., Mantel, K.-H., \& Horne, K. 1997, A\& Ap, 319, 894

Szkody, P., et al. 2005, AJ, 129, 2386

Szkody, P., et al. 2006, ApJ (Letters), 646, L147

$\dagger$ Changing $\mathrm{M}_{1}$ within reasonable limits does not significantly affect the Roche lobe size in this $\log$ scale. 